\title{
RELAÇÃO ENTRE OS RANKINGS DE SUSTENTABILIDADE E FINANCEIRO TRADICIONAIS DAS EMPRESAS CANDIDATAS AO ISE: UMA APLICAÇÃO DOS MÉTODOS DISPLACED IDEAL E DISPLACED IDEAL MODIFICADO
}

\author{
Nayane Thais Krespi \\ Universidade Federal do Paraná - UFPR \\ nkrespi@gmail.com \\ Nelson Hein \\ Universidade Regional de Blumenau - FURB \\ hein@furb.br \\ Adriana Kroenke \\ Universidade Regional de Blumenau - FURB \\ akroenke@furb.br \\ Josué Ervin Musial \\ Universidade Federal do Paraná - UFPR \\ ajmusial@gmail.com

\section{RESUMO}

Este estudo objetiva avaliar o grau de relacionamento entre os rankings formados pelos indicadores de sustentabilidade e indicadores financeiros das empresas candidatas ao Índice de Sustentabilidade Empresarial - ISE, por meio dos métodos displaced ideal e displaced ideal modificado. Na parte metodológica foram utilizados 22 indicadores de sustentabilidade e 10 indicadores financeiros, de 21 empresas diferentes listadas na BM\&FBovespa. Por fim, calculou-se o coeficiente de correlação de Kendall para determinar o quanto um ranking tinha relação com outro, em que não foram encontrados resultados significativos para nenhum dos rankings elaborados.

Palavras-chave: Ranking. Indicadores de sustentabilidade. Indicadores financeiros.

\begin{abstract}
This study aims to evaluate the degree of relationship between the rankings formed by sustainability indicators and financial indicators of the companies applying for the Corporate Sustainability Index - ISE, by the methods displaced ideal and ideal modified displaced. The theoretical part were used 22 indicators of sustainability and financial indicators 10 of 21 different companies listed on the BM \& FBovespa. Finally, we calculated the Kendall correlation coefficient to determine how much a ranking was related to another, they were not found significant results for any of the elaborate rankings.
\end{abstract}

Keywords: Ranking. Sustainability indicators. Financial indicators. 


\section{INTRODUÇÃO}

As discussões e preocupações referentes às questões ambientais atualmente têm crescido cada vez mais, visto que as exigências legais, sociais e de mercado tem se alterado também em função das novas necessidades ambientais. Esse interesse crescente provoca alterações no cenário do planejamento empresarial, que antes possuía foco somente econômico e, hoje vem trocando sua visão "antiga" para um conceito mais amplo, o de desenvolvimento sustentável (STROBEL; CORAL; SELIG, 2004).

Por sua vez, a sustentabilidade empresarial surgiu como consequência de trabalhos realizados com as comunidades, dessa mesma forma emergiu também, diversos indicadores de sustentabilidade. Nesse período temas como globalização, contabilidade aliada a cidadania, governança corporativa tornaram-se preeminentes em discussões e gestão e planejamento empresariais (STROBEL; CORAL; SELIG, 2004).

A partir desse período a sustentabilidade passou a contemplar três aspectos: econômico, ambiental e sociocultural. A estratégia ambiental presente nas empresas deve harmonizar os objetivos referentes aos três aspectos, bem como otimizar o equilíbrio entre eles. Dessa forma são atividades inerentes as empresas sustentáveis práticas como sustentar-se e expandir-se economicamente; aumento do valor para o acionista, do prestígio e da reputação; adoção de práticas consideradas éticas de negociação; criação de valores para os diferentes stakehoders; qualidade dos serviços prestados e produtos, dentre outras (NUNES, 2010).

Proceder à análise do desempenho financeiro de uma empresa, de acordo com Iudícibus (2007) é a arte de conseguir extrair relações úteis dos relatórios contábeis a fim de obter informações de qualidade acerca da situação da empresa. Entretanto o mesmo autor ainda chama atenção para o fato de que um mesmo conjunto de indicadores pode levar a diferentes conclusões tiradas por diferentes pessoas, mas quando bem utilizados, desempenham bem seu papel.

Para tanto o objetivo deste estudo é avaliar o grau de relacionamento entre os rankings formados pelos indicadores de sustentabilidade e indicadores financeiros, das empresas candidatas ao ISE, por meio dos métodos displaced ideal e displaced ideal modificado.

\section{SUSTENTABILIDADE}

Impactos oriundos do crescente desenvolvimento industrial, das forças de mercado sobre o ambiente natural, do crescimento populacional e da concentração de capital, ocasionaram crises de complexa resolução durante o século XX (MAIA; PIRES, 2011). Foi nesse contex to que surgiram as primeiras discussões acerca do tema sustentabilidade.

Ainda conforme os mesmos autores foram os excessos do sistema capitalista de produção (iniciados no século XX) sobre o ambiente natural acrescidos dos impactos negativos gerados na sociedade e percebidos por alguns países, que fizeram com que a Organização das Nações Unidas (ONU) marcasse os primeiros debates em torno do conceito de desenvolvimento sustentável, por volta de 1987.

Entretanto, Carroll já definia sustentabilidade em 1979 como as expectativas econômicas, legais éticas e discricionárias que a sociedade tem em relação as empresas por determinado período. A palavra discricionária é utilizada neste sentido para designar uma restituição à sociedade de parte do que a empresa recebeu.

Neste sentido, Bellen (2005) pondera que o conceito de sustentabilidade (desenvolvimento sustentável) é resultado de um longo processo de reavaliação da relação existente entre sociedade civil e o meio natural. O processo de desenvolvimento sustentável é contínuo e complexo, fazendo emergir uma ampla variedade de abordagens para o tema.

Existem diversas definições para sustentabilidade, sendo que uma das mais conhecidas é do Relatório Brundtland, elaborado pela World Comission on Environment and Developmet 
(WCED). Este documento define sustentabilidade como o atendimento as necessidades das gerações presentes sem comprometer a possibilidade das gerações futuras atenderem suas próprias necessidades (WCED, 1987).

Na visão de Constanza, Daly e Bartholomew (1991) o conceito de sustentabilidade precisa ser entendido dentro da relação dinâmica existente entre o sistema econômico humano e um sistema maior, o sistema ecológico. Este último, conforme o autor, é um sistema que possui uma taxa de mudança mais lenta, se comparado ao sistema econômico humano.

Dentro dessa relação, sustentabilidade deve ser entendida como a garantia de que a vida humana continue indefinidamente, com o desenvolvimento e aprimoramento cultural, de forma a considerar que os efeitos das atividades humanas permaneçam no limite das fronteiras adequadas, não destruindo a diversidade, nem a complexidade e nem as funções do sistema ecológico de suporte à vida (CONSTANZA; DALY; BARTHOLOMEW, 1991).

Goldsmith (1995) definiu que uma sociedade deve ser considerada sustentável quando se atende todos os propósitos e intenções de forma indefinida, satisfazendo por completo seus membros.

Algumas organizações não governamentais, bem como para os Programas das Nações Unidas para o Meio Ambiente e para o Desenvolvimento (Pnuma e Pnud) definem sustentabilidade como a modificação da biosfera e a aplicação de seus recursos para atender às necessidades humanas, de forma a aumentar sua qualidade de vida (IUCN, 1980).

Bellen (2005) ressalta que é necessário contemplar a sustentabilidade sob diversos aspectos, não somente pelo aspecto ambiental. Ele destaca os aspectos sociais, ambientais e econômicos. $\mathrm{O}$ autor ainda chama atenção para o fato de a sustentabilidade ter perspectivas que devem ser atendidas a curto, médio e longo prazo.

\section{AVALIAÇÃO DE DESEMPENHO POR MEIO DE INDICADORES FINANCEIROS}

Segundo Antunes e Martins (2007), dissertar sobre avaliação de desempenho não é simples, dada a abrangência do tema. Ainda de acordo com os autores, é um tema complexo por apresentar diversas metodologias eficazes de mensuração do desempenho.

A avaliação de desempenho pode ser considerada indispensável para manter a competitividade entre as empresas, visto que com o advento da tecnologia da informação e com a crescente globalização, a competitividade entre as empresas tem se tornado mais acirrada. Essa alta na competitividade faz com que as empresas busquem se diferenciar de suas concorrentes, tornando-se mais atrativa em determinados aspectos (WERNKE; LEMBECK, 2004 e PEREZ; FAMÁ, 2003).

Conforme definido por Williams (2002) avaliação de desempenho é o estabelecimento de medidas chaves, cuja função é informar aos gestores, independentemente de posição ocupada na empresa, em que nível os objetivos traçados para a empresa estão sendo atingidos. O autor ainda destaca que este tipo de avaliação é importante para a empresa, frente ao papel de informar os gestores que desenvolve.

De acordo com Miranda et al (2003), medir o desempenho de uma empresa se faz necessário, visto que esta medição deve ser fonte de informação para os gestores acerca do que está acontecendo naquele ambiente. Ainda segundo os autores, o grande problema não está em definir precisamente o que é avaliação de desempenho, e sim, em estabelecer o que deverá ser medido, para garantir a qualidade da informação.

A utilização de indicadores para avaliar o desempenho financeiro das empresas foi, para Iudícibus (2007), um grande avanço da contabilidade. O autor considera esse fato, sob o argumento de que este tipo de análise tem capacidade de retratar situações passadas e presentes, e permite inferir informações acerca de um futuro próximo.

\section{MÉTODO DISPLACED IDEAL E MÉTODO DISPLACED IDEAL MODIFICADO}


O método displaced ideal surge a partir das ideias que rodeiam a teoria Multiple Criteria Decision Making (MCDM), sendo especificamente enquadrado no modelo racional desta teoria. O principal conceito contido no método é a da solução ideal. Entretanto, durante muitos anos esse conceito foi disfarçado por diversos outros nomes (ZELENY, 1976).

A ideia de solução ideal surge no início dos anos sessenta, com o objetivo de auxiliar na tomada de decisões multiobjetivo. Logo passa a ser um conceito móvel, ou seja, não necessariamente precisa existir uma solução ideal que sempre estará no mesmo lugar (ZELENY, 1982).

Por volta de 1973, 1974 Zeleny apresentou o método por ele desenvolvido, chamado de displaced ideal, cujo conceito principal era justamente a solução ideal. Nesse momento o criador do método afirma que seu conceito já é geral o suficiente para auxiliar no processo de tomada de decisão (ZELENY, 1982).

Zeleny (1982) afirma que alguns conceitos-chave precisam ser explicados, para que posteriormente o modelo possa ser compreendido. Inicialmente conceitua-se o conjunto $X=$ $\left\{x^{1}, x^{2}, \ldots, x^{m}\right\}$ como sendo o conjunto de alternativas inicialmente viáveis. Nesse sentido, cada alternativa é caracterizada por $n$ atributos. Por exemplo, a $k$-ésima alternativa pode ser representada por:

$$
x^{k}=\left(x_{1}^{k}, x_{2}^{k}, \ldots, x_{n}^{k}\right) \quad k=1, \ldots, m
$$

Individualmente, $x_{i}^{k}$ indica o nível do atributo i atingido pela alternativa $\mathrm{k}$, em que $i=$ $1, \ldots, n ; k=1, \ldots, m$.

Cabe ressaltar que neste ponto, Zeleny (1982) chama atenção para a diferença entre atributos e objetivos e critérios. Na visão de Zeleny, atributos dizem respeito a características físicas e fisiológicas, pontuações que podem ser obtidas por meio de medição objetiva (cavalos de potência por exemplo). Ao passo que os critérios ou objetivos são classificados como medidas obtidas de formas subjetivas (prestígio e imagem, por exemplo).

Entretanto, para facilitar a compreensão, há de se uniformizar a nomenclatura durante a exposição do método. Ou seja, tratar-se-á sempre de atributos (independentemente de ser atributo ou critério) (ZELENY, 1982).

Dessa forma, $x^{k}$ é simplesmente um vetor de $n$ números, atribuído a cada $x$ e resume as informações disponíveis sobre $x^{k}$ em termos quantitativos e qualitativos, objetivos e subjetivos, atributos e critérios. Estabeleceu-se assim a chamada alternativa de multiatributos. Analisando o $i$-ésimo atributo individualmente, verifica-se o conjunto $X$ que gera $m$ números, representados por um vetor que evidencia o nível do atributo $i$.

$$
x_{i}=\left(x_{i}^{1}, \ldots, x_{i}^{m}\right)
$$

Sua interpretação mais simples é sempre assumir que um é sempre preferido em detrimento do outro, ou vice-versa. Pois,

$$
\operatorname{Min}_{k} x_{i}^{k}=\operatorname{Max}_{k}\left(-x_{i}^{k}\right) \quad k=1,2, \ldots, m
$$

É importante destacar que encontrar o mínimo de $m$ números é o mesmo que encontrar o máximo de $m$ com sinal negativo. Essa relação é importante, pois para exposição do método, todos os critérios serão determinados pelo valor máximo. Todavia, conforme afirma Zeleny (1976), em problemas reais, nem sempre é o valor máximo que se quer. 
Dentre todos os resultados alcançáveis para qualquer atributo i, conforme vetor $x_{i}$, há pelo menos um valor extremo (ou ideal) que é preferível a todos os demais. Este valor é chamado de "valor âncora", representado por $x_{i}^{*}$. Logo,

$$
x_{i}^{*}=\operatorname{Max}_{k} x_{i}^{k} \quad i=1,2, \ldots, n
$$

Ressalta-se que a operação Max (acima) é uma simplificação, pois ambos os valores, máximo é ideal, estão abarcados pelo valor âncora. O conjunto composto por todos os valores âncoras serão chamado de alternativa ideal, e representados por:

$$
x^{*}=\left(x_{1}^{*}, \ldots, x_{n}^{*}\right)
$$

Nesse sentido, o ideal desempenha papel de destaque no processo de tomada de decisões. Por exemplo, supõe-se a existência de $x^{k}$ tal que $x^{k} \equiv x^{*}$, a alternativa ideal é atingível pela escolha do $x^{k}$. Nesse caso, não existe decisão para se tomar. Qualquer função concebível (e racional) tem sua utilidade definida sobre $n$-tupla de números $\left(x_{1}, \ldots, x_{n}\right)$, que atingiria valor máximo em $x^{*}$ e consequentemente em $x^{k}$.

Portanto, o ideal não é, contudo, possível em geral, ou se possível, logo se torna inviável em função de o tomador de decisão considerar apenas um $x_{i}$ (ZELENY, 1982).

\section{PROCEDIMENTOS METODOLÓGICOS}

A população de pesquisa compreende todas as empresas elegíveis ao ISE 2011/2012, perfazendo um total e 182 empresas. Compõem esse grupo empresas pertencentes aos diversos setores econômicos da BM\&FBovespa.

Essas empresas foram escolhidas para compor a pesquisa, em função, inicialmente, de também serem candidatas a compor o ISE, possibilitando comparações posteriores. Num segundo momento percebe-se a relevância da amostra também por serem essas as empresas com as ações mais líquidas negociadas na bolsa de valores (Processo Seletivo 2011-2012 / Informações Gerais, 2011).

Sendo assim, a amostra de pesquisa foi definida como sendo as empresas listadas na BM\&FBovespa, candidatas ao ISE, que apresentavam documentos específicos de sustentabilidade e que possuam os indicadores utilizados na pesquisa divulgados nesses documentos. Ao final dessa seleção sobraram 24 empresas, entretanto 3 delas pertencem ao setor financeiro e foram excluídas também.

Portanto, a amostra da pesquisa é composta por 21 empresas, também pertencentes a diferentes setores econômicos na BM\&FBovespa exceto ao setor financeiro. Cabe ressaltar que são 24 empresas, de diferentes setores de atuação, visto que apenas estas apresentavam os dados necessários para a realização da pesquisa na data da coleta.

\subsection{COLETA E ANÁLISE DOS DADOS}

Para realizar a coleta dos dados de sustentabilidade, foi necessário baixar os relatórios de sustentabilidade, referentes ao ano de 2010, disponibilizados pelas empresas componentes da amostra, em seu respectivo sítio eletrônico. As empresas que não possuíam esse documento disponível na data da coleta foram automaticamente excluídas da amostra da pesquisa.

Destaca-se que, como no Brasil a divulgação de informações sustentáveis não é obrigatória e nem padronizada, apenas 22 dos 70 indicadores possuíam informações divulgadas. Alguns indicadores ainda, não apresentam caráter quantitativo, sendo inviável a utilização destes na presente pesquisa. 
Sendo assim, foram coletadas 22 informações referentes à sustentabilidade diferentes para cada uma das empresas componentes da amostra, sendo 4 indicadores econômicos, 7 indicadores ambientais, 5 indicadores de práticas trabalhistas e dignidade no trabalho, 2 indicadores de direitos humanos, 2 indicadores sociais e 2 indicadores de responsabilidade pelo produto.

Para obtenção dos dados necessários para determinação dos indicadores financeiros, utilizou-se o software Economática. Os dados coletados referem-se ao ano de 2010 (mesmo período base dos indicadores sustentáveis).

Inicialmente procedeu-se a normalização dos dados coletados, tanto os dados de sustentabilidade quanto os dados financeiros. Para tanto, utilizou-se a seguinte métrica:

$$
x_{i_{N}}^{k}=\frac{x_{i}^{k}}{\sqrt[2]{\left(x_{i}^{1}\right)^{2}+\left(x_{i}^{2}\right)^{2}+\ldots+\left(x_{i}^{k}\right)^{2}}}
$$

Na sequência, efetuou-se o cálculo das distâncias entre os cenários reais apresentados pelas empresas e o cenário ideal, utilizando a métrica de Mahalonobis, para os indicadores de sustentabilidade. As medidas de distância foram calculadas com o auxílio dos softwares MatLab e LHStat 2.0. Posteriormente foram elaborados, por meio do método Displaced Ideal Modificado, o ranking de sustentabilidade das empresas.

\section{APRESENTAÇÃO E ANÁLISE DOS RESULTADOS}

A Tabela 1 apresenta o ranking geral de sustentabilidade e financeiro, determinado pelas distâncias relativas ao meio e calculado pelo método Displaced Ideal, ou seja, com a aplicação da métrica Euclidiana.

Tabela 1 - Ranking geral de sustentabilidade e financeiro - DRM - Método Displaced Ideal

\begin{tabular}{l|l|c|l|c}
\hline \multirow{2}{*}{ Posição } & \multicolumn{2}{|c|}{ Sustentabilidade } & \multicolumn{2}{c}{ Financeiro } \\
\cline { 2 - 5 } & \multicolumn{1}{c|}{ Empresas } & DRM - Média & \multicolumn{1}{c}{ DRM - Média } \\
\hline $1^{\circ}$ & PETROBRÁS & 0.552 & ETERNIT & 0.536 \\
\hline $2^{\circ}$ & TRACTEBEL & 0.544 & ELETROBRAS & 0.399 \\
\hline $3^{\circ}$ & COMGAS & 0.543 & PETROBRÁS & 0.388 \\
\hline $4^{\circ}$ & P.ACUCAR-CBD & 0.541 & USIMINAS & 0.385 \\
\hline $5^{\circ}$ & KLABIN S/A & 0.539 & TIM PART S/A & 0.333 \\
\hline $6^{\circ}$ & EMBRAER & 0.538 & DURATEX & 0.296 \\
\hline $7^{\circ}$ & CEMIG & 0.536 & EVEN & 0.274 \\
\hline $8^{\circ}$ & INDS ROMI & 0.533 & KLABIN S/A & 0.268 \\
\hline $9^{\circ}$ & USIMINAS & 0.527 & SUZANO PAPEL & 0.261 \\
\hline $10^{\circ}$ & EVEN & 0.523 & INDS ROMI & 0.243 \\
\hline $11^{\circ}$ & ETERNIT & 0.521 & COMGAS & 0.237 \\
\hline $12^{\circ}$ & SUZANO PAPEL & 0.510 & BRF FOODS & 0.235 \\
\hline $13^{\circ}$ & ENERGIAS BR & 0.509 & EMBRAER & 0.209 \\
\hline $14^{\circ}$ & ECORODOVIAS & 0.501 & ECORODOVIAS & 0.203 \\
\hline $15^{\circ}$ & FIBRIA & 0.490 & FIBRIA & 0.200 \\
\hline $16^{\circ}$ & NATURA & 0.490 & TRACTEBEL & 0.182 \\
\hline $17^{\circ}$ & BRASKEM & & 0.180 \\
\hline
\end{tabular}




\begin{tabular}{l|l|l|l|l}
$18^{\circ}$ & DURATEX & 0.474 & ENERGIAS BR & 0.173 \\
\hline $19^{\circ}$ & ELETROBRAS & 0.452 & P.ACUCAR-CBD & 0.171 \\
\hline $20^{\circ}$ & BRF FOODS & 0.400 & CEMIG & 0.152 \\
\hline $21^{\circ}$ & TIM PART S/A & 0.273 & BRASKEM & 0.108 \\
\hline
\end{tabular}

Fonte: dados da pesquisa

Segundo o exposto na Tabela 1 a empresa Petrobrás é a mais sustentável dentre as pesquisadas, porém no ranking financeiro passa a ser a $4^{\circ}$ colocada. Pode-se afirmar que essa empresa é $71.8 \%$ sustentável em relação ao máximo que poderia ser, nesse mesmo sentido, ela é 38.5\% no desempenho financeiro em relação ao máximo que poderia ser.

Atenta-se para o fato de esta empresa pertencer ao Mercado Tradicional e também não fazer parte do ISE, fazendo com que esse resultado seja inusitado de acordo com a literatura. . Logo, supõe-se que esse resultado seja devido ao tamanho da empresa e ao seu caráter estatal, o que a faz desenvolver vários projetos socais, patrocinar diferentes atividades, ter cuidados com o meio ambiente, entre outros.

Em seguida, observa-se a Tractebel ocupando $2^{\circ}$ lugar no ranking de sustentabilidade e $17^{\circ}$ lugar no ranking financeiro. De acordo com o ranking. A Tractebel é uma empresa extremamente preocupada com o desenvolvimento sustentável, porém nem tão eficiente sob a ótica financeira. Dado o fato de esta empresa pertencer ao Novo Mercado e integrar o ISE, já era de se esperar que ela fosse desenvolvida sustentavelmente.

Destaca-se também a Natura que ficou em $1^{\circ}$ lugar no ranking financeiro, mas apenas em $16^{\circ}$ no de sustentabilidade. Neste sentido, pondera-se que essa empresa possui um bom desempenho financeiro, entretanto não se pode dizer o mesmo em relação às práticas de sustentabilidade por ela desenvolvidas. E esse comportamento desigual entre a posição ocupada no ranking de sustentabilidade e no ranking financeiro pode ser verificado em diversas outras empresas, como por exemplo, a Cemig, a Tim Part S/A, a Duratex, dentre outras.

Ainda de acordo com a Tabela 1, a Braskem foi a última colocada no ranking financeiro e a $17^{\mathrm{a}}$ colocada no ranking de sustentabilidade, caracterizando-se, portanto como uma das piores empresas analisadas. De forma geral, observou-se que as empresas que são melhores em sustentabilidade não o são nos aspectos financeiros e vice-versa. Contudo é necessário ponderar que existem algumas empresas que se mantém estável em ambos os rankings, como por exemplo, a Petrobrás e a Braskem.

Na Tabela 2 encontra-se o ranking geral de sustentabilidade e financeiro, determinado pelas distâncias relativas ao grupo e calculado pelo método Displaced Ideal, logo, foi utilizada a métrica Euclidiana.

Tabela 2- Ranking geral de sustentabilidade e financeiro - DRG - Método Displaced Ideal

\begin{tabular}{c|l|c|l|c}
\hline \multirow{2}{*}{ Posição } & \multicolumn{2}{|c|}{ Sustentabilidade } & \multicolumn{2}{c}{ Financeiro } \\
\cline { 2 - 5 } & \multicolumn{1}{|c|}{ Empresas } & DRG - Média & \multicolumn{1}{c}{ Empresas } & DRG - Média \\
\hline $1^{\mathbf{o}}$ & PETROBRÁS & 0.9090 & NATURA & 0.5718 \\
\hline $2^{\mathbf{o}}$ & TRACTEBEL & 0.6268 & PETROBRÁS & 0.4861 \\
\hline $3^{\mathbf{o}}$ & COMGAS & 0.6178 & ELETROBRAS & 0.4409 \\
\hline $4^{\mathbf{o}}$ & CEMIG & 0.6118 & ETERNIT & 0.4198 \\
\hline $5^{\mathbf{o}}$ & P.ACUCAR-CBD & 0.6109 & USIMINAS & 0.3514 \\
\hline $6^{\mathbf{o}}$ & EMBRAER & 0.6083 & TIM PART S/A & 0.3256 \\
\hline $7^{\mathbf{o}}$ & KLABIN S/A & 0.6060 & DURATEX & 0.2808 \\
\hline $8^{\circ}$ & INDS ROMI & 0.5908 & KLABIN S/A & 0.2702 \\
\hline
\end{tabular}




\begin{tabular}{c|l|l|l|l}
$9^{\circ}$ & USIMINAS & 0.5827 & EVEN & 0.2675 \\
\hline $10^{\circ}$ & SUZANO PAPEL & 0.5744 & SUZANO PAPEL & 0.2500 \\
\hline $11^{\circ}$ & EVEN & 0.5690 & INDS ROMI & 0.2429 \\
\hline $12^{\circ}$ & ETERNIT & 0.5623 & COMGAS & 0.2292 \\
\hline $13^{\circ}$ & ENERGIAS BR & 0.5378 & BRF FOODS & 0.2233 \\
\hline $14^{\circ}$ & BRASKEM & 0.5318 & EMBRAER & 0.2081 \\
\hline $15^{\circ}$ & ECORODOVIAS & 0.5300 & FIBRIA & 0.1869 \\
\hline $16^{\circ}$ & ELETROBRAS & 0.5059 & P.ACUCAR-CBD & 0.1846 \\
\hline $17^{\circ}$ & DURATEX & 0.4990 & ECORODOVIAS & 0.1794 \\
\hline $18^{\circ}$ & FIBRIA & 0.4981 & TRACTEBEL & 0.1736 \\
\hline $19^{\circ}$ & NATURA & 0.4493 & ENERGIAS BR & 0.1712 \\
\hline $20^{\circ}$ & BRF FOODS & 0.4387 & CEMIG & 0.1471 \\
\hline $21^{\circ}$ & TIM PART S/A & 0.3116 & BRASKEM & 0.1039 \\
\hline
\end{tabular}

Fonte: dados da pesquisa

Conforme indicado na Tabela 2 a empresa Petrobrás é a mais sustentável dentre as pesquisadas, como também é quando analisada pela distância relativa ao meio. Porém no ranking financeiro passa a ser a $2^{\circ}$ colocada, sendo melhor do que na análise pela distância relativa ao meio. A Petrobrás é, portanto, $90.90 \%$ próxima ao melhor cenário do grupo nos aspectos sustentáveis e $48.61 \%$ próxima ao melhor cenário financeiro do grupo.

Também como nos rankings baseados nas distâncias relativas ao meio, a Petrobrás é seguida pela empresa Tractebel, sendo esta a $2^{\circ}$ no quesito sustentabilidade. Entretanto, a empresa para $18^{\circ}$ lugar quando analisada sob os aspectos financeiros, sendo uma posição a mais do que no ranking anterior.

A empresa Braskem, permanece sendo a última colocada no ranking financeiro, mas passa a ser $14^{\circ}$ colocada no ranking de sustentabilidade. Por sua vez a natura continua sendo a melhor empresa sob a ótica financeira, contudo é somente a $19^{\circ}$ colocada no quesito sustentabilidade, evidenciando ainda mais essa diferença.

No geral, é possível concluir de forma semelhante aos rankings elaborados com base nas distâncias relativas ao meio. Já foram elencadas algumas sutis diferenças, todavia de as empresas mais sustentáveis ainda apresentam um pior desempenho financeiro em relação àquelas que não são desenvolvidas sustentavelmente. Algumas exceções ainda existem, como a Petrobrás e a Braskem.

A Tabela 3 expõe o ranking geral de sustentabilidade e financeiro, elaborado a partir das distâncias relativas ao meio, determinadas pela métrica de Mahalanobis, logo este é o ranking criado a partir do método Displaced Ideal Modificado.

Tabela 3 - Ranking geral de sustentabilidade e financeiro - DRM - Método Displaced Ideal Modificado

\begin{tabular}{c|l|c|l|c}
\hline \multirow{2}{*}{ Posição } & \multicolumn{2}{|c|}{ Sustentabilidade } & \multicolumn{2}{c}{ Financeiro } \\
\cline { 2 - 5 } & \multicolumn{1}{|c|}{ Empresas } & DRM - Méida & \multicolumn{1}{c}{ Empresas } & DRM - Média \\
\hline $1^{\text {o }}$ & PETROBRÁS & 0.665 & NATURA & 0.587 \\
\hline $2^{\circ}$ & TRACTEBEL & 0.558 & ETERNIT & 0.407 \\
\hline $3^{\circ}$ & INDS ROMI & 0.534 & ELETROBRAS & 0.404 \\
\hline $4^{\circ}$ & COMGAS & 0.531 & PETROBRÁS & 0.361 \\
\hline $5^{\circ}$ & NATURA & 0.525 & USIMINAS & 0.342 \\
\hline $6^{\circ}$ & EMBRAER & 0.515 & DURATEX & 0.317 \\
\hline $7^{\circ}$ & KLABIN S/A & 0.510 & KLABIN S/A & 0.263 \\
\hline
\end{tabular}




\begin{tabular}{l|l|l|l|l}
$8^{\circ}$ & USIMINAS & 0.508 & TIM PART S/A & 0.263 \\
\hline $9^{\circ}$ & P.ACUCAR-CBD & 0.507 & COMGAS & 0.260 \\
\hline $10^{\circ}$ & CEMIG & 0.505 & EVEN & 0.255 \\
\hline $11^{\circ}$ & EVEN & 0.502 & SUZANO PAPEL & 0.250 \\
\hline $12^{\circ}$ & ENERGIAS BR & 0.500 & BRF FOODS & 0.240 \\
\hline $13^{\circ}$ & SUZANO PAPEL & 0.498 & INDS ROMI & 0.230 \\
\hline $14^{\circ}$ & FIBRIA & 0.496 & ENERGIAS BR & 0.223 \\
\hline $15^{\circ}$ & ETERNIT & 0.490 & FIBRIA & 0.218 \\
\hline $16^{\circ}$ & ECORODOVIAS & 0.489 & TRACTEBEL & 0.213 \\
\hline $17^{\circ}$ & DURATEX & 0.479 & ECORODOVIAS & 0.200 \\
\hline $18^{\circ}$ & BRASKEM & 0.430 & EMBRAER & 0.189 \\
\hline $19^{\circ}$ & BRF FOODS & 0.404 & P.ACUCAR-CBD & 0.188 \\
\hline $20^{\circ}$ & ELETROBRAS & 0.394 & CEMIG & 0.154 \\
\hline $21^{\circ}$ & TIM PART S/A & 0.275 & BRASKEM & 0.130 \\
\hline
\end{tabular}

Fonte: dados da pesquisa

A Tabela 3 evidencia que a empresa Petrobrás foi a mais sustentável dentre as pesquisadas e a $4^{\circ}$ melhor nos aspectos financeiros. Esse resultado corrobora com o ranking obtido pelo método displaced ideal baseado nas distâncias relativas ao meio, visto que as posições ocupadas pela Petrobrás são as mesmas em ambos.

Ainda corroborando com o mesmo ranking elaborado pelo método displaced ideal, a empresa Tractebel é a $2^{\circ}$ mais sustentável e somente a $16^{\circ}$ nos aspectos financeiros. Diferentemente do ranking criado pelo método displaced ideal, neste a empresa Natura passa a ocupar o $5^{\circ}$ lugar no ranking de sustentabilidade e se mantém em $1^{\circ}$ no ranking financeiro.

Destaque para a Braskem, classificada como a pior no desempenho financeiro e como a $18^{\circ}$ pior no quesito sustentabilidade. Essa empresa também, conforme os rankings elaborados pelo outro método, é uma das piores dentre as analisadas.

De forma geral não é possível verificar muitas alterações nos rankings criados pelo método displaced ideal para o elaborado pelo método displaced ideal modificado, sendo ambos baseados nas distâncias relativas ao meio. Porém é possível observar, uma tendência de que as empresas ranqueadas pelo segundo método sejam mais estáveis em relação aosdois quesitos analisados.

A Tabela 4 evidencia o ranking geral de sustentabilidade e financeiro, elaborado a partir das distâncias relativas ao grupo, determinadas pela métrica de Mahalanobis. Nesse caso, portanto este é o ranking criado a partir do método Displaced Ideal Modificado.

Tabela 4 - Ranking geral de sustentabilidade e financeiro - DRG - Método Displaced Ideal Modificado

\begin{tabular}{c|l|l|l|c}
\hline \multirow{2}{*}{ Posição } & \multicolumn{2}{|c|}{ Sustentabilidade } & \multicolumn{2}{c}{ Financeiro } \\
\cline { 2 - 5 } & \multicolumn{1}{|c|}{ Empresas } & DRG & \multicolumn{1}{c}{ Empresas } & DRG \\
\hline $1^{\mathbf{o}}$ & PETROBRÁS & 0.866 & NATURA & 0.704 \\
\hline $2^{\mathbf{0}}$ & TRACTEBEL & 0.645 & PETROBRÁS & 0.520 \\
\hline $3^{\mathbf{0}}$ & COMGAS & 0.624 & ETERNIT & 0.504 \\
\hline $4^{\mathbf{0}}$ & INDS ROMI & 0.621 & ELETROBRAS & 0.492 \\
\hline $5^{\mathbf{0}}$ & CEMIG & 0.584 & USIMINAS & 0.426 \\
\hline $6^{\mathbf{0}}$ & EMBRAER & 0.578 & DURATEX & 0.383 \\
\hline $7^{\circ}$ & SUZANO PAPEL & 0.577 & TIM PART S/A & 0.365 \\
\hline $8^{\mathbf{0}}$ & ENERGIAS BR & 0.547 & KLABIN S/A & 0.324 \\
\hline
\end{tabular}




\begin{tabular}{c|l|l|l|l}
$9^{\circ}$ & KLABIN S/A & 0.539 & BRF FOODS & 0.320 \\
\hline $10^{\circ}$ & NATURA & 0.529 & SUZANO PAPEL & 0.316 \\
\hline $11^{\circ}$ & DURATEX & 0.525 & FIBRIA & 0.279 \\
\hline $12^{\circ}$ & P.ACUCAR-CBD & 0.523 & EVEN & 0.279 \\
\hline $13^{\circ}$ & EVEN & 0.497 & ENERGIAS BR & 0.278 \\
\hline $14^{\circ}$ & FIBRIA & 0.490 & INDS ROMI & 0.274 \\
\hline $15^{\circ}$ & USIMINAS & 0.483 & COMGAS & 0.272 \\
\hline $16^{\circ}$ & BRF FOODS & 0.481 & P.ACUCAR-CBD & 0.264 \\
\hline $17^{\circ}$ & ECORODOVIAS & 0.470 & TRACTEBEL & 0.255 \\
\hline $18^{\circ}$ & ETERNIT & 0.464 & EMBRAER & 0.235 \\
\hline $19^{\circ}$ & ELETROBRAS & 0.344 & ECORODOVIAS & 0.190 \\
\hline $20^{\circ}$ & BRASKEM & 0.342 & CEMIG & 0.186 \\
\hline $21^{\circ}$ & TIM PART S/A & 0.329 & BRASKEM & 0.180 \\
\hline
\end{tabular}

Fonte: dados da pesquisa

Segundo as informações expostas na Tabela 4, a empresa Braskem continua sendo a pior sob a ótica financeira, exatamente como no ranking referente às distâncias relativas ao meio. Entretanto, quando analisada sob o aspecto da sustentabilidade a empresa passa a ser somente $32.9 \%$ próxima ao melhor desempenho do grupo, caindo, dessa forma para a $20^{\circ}$ posição.

A Petrobrás continua em destaque por ser a empresa mais sustentável dentre as analisadas e por apresentar o $2^{\circ}$ melhor desempenho financeiro. Este é o mesmo resultado obtido com os rankings baseados nas distâncias relativas ao grupo, elaborados a partir do método displaced ideal.

Generalizando, os resultados obtidos neste ranking, são bastante semelhantes àqueles encontrados nos rankings baseados nas distâncias relativas ao meio. Esse resultado já era esperado, visto que existe ligação entre as duas medidas. Nesse mesmo sentido, também é possível ponderar que ao variar o método de elaboração dos rankings, apenas sutis diferenças foram observadas, não sendo essas suficientemente fortes para alterar as considerações finais.

\subsection{COEFICIENTE DE CORRELAÇÃO DE KENDALL}

A Tabela 5 expõe o resultado obtido na correlação de Kendall.

Tabela 5 - Coeficiente de correlação de Kendall

\begin{tabular}{l|c|c|c|c} 
& $\begin{array}{c}\text { DRM - Displaced } \\
\text { Ideal }\end{array}$ & $\begin{array}{c}\text { DRM - Displaced } \\
\text { Ideal Modificado }\end{array}$ & $\begin{array}{c}\text { DRG - Displaced } \\
\text { Ideal }\end{array}$ & $\begin{array}{c}\text { DRG - Displaced } \\
\text { Ideal Modificado }\end{array}$ \\
\hline Coeficiente & -0.124 & 0.029 & -0.181 & -0.171 \\
\hline Significância & 0.432 & 0.856 & 0.251 & 0.277 \\
\hline
\end{tabular}

Fonte: dados da pesquisa

Conforme evidenciado na Tabela 5, não se constatou correlação significante entre os rankings de sustentabilidade e financeiro calculados pelo método displaced ideal, tanto com base nas distâncias relativas ao meio, como baseados nas distâncias relativas ao grupo. Ainda segundo a Tabela 5, os rankings de sustentabilidade e financeiro elaborados pelo método displaced ideal modificado, tanto com as distâncias relativas ao meio quanto com as distâncias relativas ao grupo, também não apresentaram correlação significativa.

Ressalta-se que esses resultados vão ao encontro daqueles obtidos por Farias (2008) em seu estudo. Que também não evidenciou relação estatisticamente significativa entre o desempenho ambiental e o desempenho financeiro das empresas por ele pesquisadas. Ainda 
apresenta-se a pesquisa de Macedo et al (2008) que novamente não foi capaz de evidenciar diferença significativa entre o desempenho contábil-financeiro das empresas socialmente responsáveis e daquelas que não o são.

Nunes (2010), uma vez mais, não conseguiu estabelecer diferenças significativas entre os índices contábeis das empresas sustentáveis e daqueles que não o são. Dessa forma os achados da presente pesquisa estão na mesma linha dos trabalhos teórico-empíricos citados.

Por outro lado alguns estudos já evidenciaram resultado positivo em relação à análise de indicadores econômicos e sustentáveis não corroborando com os resultados do presente estudo. Como exemplo tem-se a pesquisa de Lee, Faff, Langfield-Smith (2009) que encontraram relação significativa e negativa entre a perfomance das empresas e os investimentos sustentáveis.

Ainda nesse mesmo sentido, expõe-se o estudo de López, Garcia, Rodiguez (2007), cujos resultados evidenciam impacto negativo no curto prazo na adoção de práticas de responsabilidade social. Todavia, os autores conseguiram comprovar que no longo prazo a situação se inverte, visto que no longo prazo o impacto passa a ser positivo.

\section{CONCLUSÕES}

Esta pesquisa teve como objetivo avaliar o grau de relacionamento entre os rankings formados pelos indicadores de sustentabilidade e indicadores financeiros, das empresas candidatas ao ISE, por meio dos métodos diplaced ideal e displaced ideal modificado.

Para tanto utilizou informações obtidas a partir dos relatórios de sustentabilidade e do software Economática, bem como consulta as demonstrações contábeis disponibilizadas no endereço eletrônico da BM\&FBovespa. Optou-se por utilizar os indicadores de sustentabilidade em conformidade com os propostos no documento G3.1 reformulado pelo GRI em 2011. Já para os indicadores financeiros utilizaram-se os resultados encontrados por Bastos (2008). No total foram utilizados 22 indicadores de sustentabilidade e 10 indicadores financeiros.

No que tange o objetivo da pesquisa, avaliar o grau de relacionamento entre os rankings formados pelos indicadores de sustentabilidade e indicadores financeiros, das empresas candidatas ao ISE, por meio dos métodos displaced ideal e displaced ideal modificado, conclui-se que não existe um grau de relacionamento significativo.

Pondera-se ainda que as empresas analisadas, em geral são melhores nos aspectos de sustentabilidade do que nos aspectos financeiros, visto que as distâncias relativas calculadas são maiores para os indicadores de sustentabilidade do que para os indicadores financeiros. Outra tendência observada é que para os rankings elaborados pelo método displaced ideal modificado as empresas parecem mais estáveis em suas posições, ou seja, as empresas que são mais sustentáveis tendem a ser melhores financeiramente. Entretanto é uma tendência, verificada pelo fato de as diferenças entre as posições serem menores para o método displaced ideal modificado.

\section{REFERÊNCIAS}

ANTUNES, M.T.P.; MARTINS, E. Capital intelectual: seu entendimento e seus impactos no desempenho de grandes empresas brasileiras. Revista de Administração e Contabilidade 
da Unisinos, v. 4, n.1, 2007.

BASTOS, E. C. Análise dos indicadores econômico-financeiros relevantes para avaliação de empresas. Dissertação (Mestrado em Ciências Contábeis). Programa de Pós-Graduação em Ciências Contábeis, FURB. Santa Catarina, 2008.

BELLEN, H. M. V. Indicadores de sustentabilidade: uma análise comparativa. 1. ed. Rio de Janeiro: Editora FGV, 2005. 253 p.

CARROLL, A. B. Corporate social responsibility: evolution of a definitional construct. Business \& Society, vol. 38, n. 3, 1999.

CONSTANZA, R.; DALY, H. E.; BARTHOLOMEW, J. A. Goals, agenda, and policy: recommendations for ecological economics. Ecological economics, p. 1-20, 1991.

FARIAS, K. T. R. A relação entre divulgação ambiental, desempenho ambiental e desempenho econômico nas empresas brasileiras de capital aberto: uma pesquisa utilizando equações simultâneas. Dissertação (Mestrado em Controladoria e Contabilidade). Departamento de Contabilidade e Atuária da Faculdade de Economia, Administração e Contabilidade, USP - Ribeirão Preto. São Paulo, 2008

GOLDSMITH, Edward. O desafio ecologico. Lisboa : Inst. Piaget, [1995]. 573p, il. (Perspectivas ecologicas, 10). Traducao de: The way, an ecological world view.

IUCN / UNEP / WWF. World Conservation Strategy: living resource conservation for sustainable development. Gland, Switzerland \& Nairobi, Kenya: International Union for Conservation of Nature Resources (IUCN), United Nations Enviroment Programme (UNEP) \& World Wildlife Found (WWF), 1980.

IUDÍCIBUS, S. de. Análise de balanços: a análise da liquidez e do endividamento, a análise do giro, a ánalise da rentabilidade, a análise da alavancagem financeira, indicadores e análises especiais (análise de tesouraria de Fleuriet, EVA, DVA e EBITDA).8. ed. rev. e atual. São Paulo : Atlas, 2007. xii, 240 p, il.

LEE, D. D.; FAFF, R. W.; LANGFIELD-SMITH, K. Revisting the vexing question: does superior corporate social performance lead to improved financial performance? Australian Journal of Management, v. 34, n.1, p. 21-49, 2009.

LÓPEZ, M. V.; GARCIA, A.; RODRIGUEZ, L. Sustainable development and corporate performance: a study based on the Dow Jones Sustainability Index. Journal of Busisnes Ethics, v. 75, p. 275-300, 2007.

MACEDO, M. A. da S.; SOUSA, A. C.; SOUSA, A. C. C.; CÍPOLA, F.C. Análise comparativa do desempenho contábil-financeiro de empresas socialmente responsáveis. Pensar Contábil, v. 11, n. 43, p. 15-23, 2008.

MAIA, A. G.; PIRES, P. dos S. Uma compreensão de sustentabilidade por meio dos níveis de complexidade das decisões organizacionais. Revista de Administração Mackenzie, v. 12, n. 3, p. 177-206, 2011.

MIRANDA, L. C.; MEIRA, J. M.; WANDERLEY, C. A.; SILVA, A. C. M. da. Indicadores de desempenho empresarial divulgados por empresas norte-americanos. Contabilidade Vista e Revista, v. 14, n. 2, p. 85-103, 2003.

NUNES, T. C. S. Indicadores contábeis como medidas de risco e retorno diferenciados de empresas sustentáveis: um estudo no mercado brasileiro. Dissertação (Mestrado em Controladoria e Contabilidade). Departamento de Contabilidade e Atuária da Faculdade de Economia, Administração e Contabilidade, USP. São Paulo, 2010.

PEREZ, M. M.; FAMÁ, R. Métodos de avaliação de empresas e o balanço de determinação. Caderno de Pesquisa em Administração, v. 10, n. 4, 2003.

STROBEL, J. S.; CORAL, E.; SELIG, P. M. Indicadores de sustentabilidade corporativa: uma análise comparativa. In: ENCONTRO ANUAL DA ANPAD, 28., Curitiba, 2004, 
Anais...Curitiba: ANPAD, 2004. CD-ROM.

TAVANA, M.; YAZDI, A. K.; SHIRI, M.; RAPPAPORT, J. An EFQM-Rembrandt excellence model based on the theory of displaced ideal. Benchmarking: An International Journal, v. 18, n. 5, p. 644-667, 2011

WCED - World Comission on Environment and Development. Our Common Future. Oxford University Press. Oxford. England. 1987.

WERNKE, R. LEMBECK, M. Análise de rentabilidade dos segmentos de mercado de empresas distribuidoras de mercadorias. Revista Contabilidade e Finanças, n. 35, p. 68-83, 2004.

WILLIAMS, Kathy. New developments in performance management. Strategic Finance, 2002.

ZELENY, M. Multiple criteria decision making Kyoto 1975. Springer-Verlag-Berlin: Heidelberg, 1976.

ZELENY, M. Multiple criteria decision making. New York: McGraw-Hill, 1982 